\title{
A NOTE ON TOPOLOGICAL FEEDBACK ENTROPY AND INVARIANCE ENTROPY
}

FRITZ COLONIUS, CHRISTOPH KAWAN

INSTITUT FÜR MATHEMATIK, UNIVERSITÄT AUGSBURG, 86135 AUGSBURG, GERMANY, AND GIRISH NAIR

DEPT. ELECTRICAL \& ELECTRONIC ENGINEERING, UNIVERSITY OF MELBOURNE, VIC 3010, AUSTRALIA

\begin{abstract}
For discrete-time control systems, notions of entropy for invariance are compared. One is based on feedbacks, the other one on open-loop control functions. Under a strong invariance condition, it is shown that they are essentially equivalent. Several modifications are also discussed.
\end{abstract}

January 29, 2013

Keywords: Topological feedback entropy, invariance entropy

1. Introduction. The purpose of this note is to analyze the relation between two entropy notions for invariance properties of control systems (we just call them control entropies, for short) which have been considered over the past years; there are also several slightly differing versions around. Hence, it appears worthwhile to us to clarify some relations between these concepts which have been introduced in order to describe minimal data rates in control systems with restricted digital communication channels.

In the paper by Nair, Evans, Mareels, and Moran [16] the notion of topological feedback entropy was introduced. A main result, [16, Theorem 1], shows that the invariance topological feedback entropy coincides with the feedback data rate using certain symbolic controllers (coder-controllers). Based on this relation, the second part of that paper shows that the minimal data rate for stabilizing systems via codercontrollers coincides with a local version of the topological feedback entropy at an equilibrium, where the control range is taken arbitrarily small.

Later, Colonius and Kawan [4] introduced invariance entropy for continuous-time control systems. In the doctoral thesis [11] by Kawan and in his subsequent papers [12, 13, 14], as well as in Colonius and Kawan [5] and Colonius and Helmke [3] this notion has been further elaborated and used to analyze properties of control systems. The forthcoming monograph [15] will give a comprehensive presentation of these results. In Da Silva [6], the notion of invariance entropy has been generalized to specific timedependent control systems, namely control systems with random dynamics modelled by a measurable dynamical system.

Both notions of control entropy go back to topological entropy in the mathematical theory of dynamical systems. They differ in the following points: the control entropy in [16] is based on feedbacks, it is defined for discrete-time systems, and it is modelled after the original definition of topological entropy due to Adler, Konheim, and McAndrew [1]. In contrast, the control entropy in [4] refers to open-loop, i.e., time-dependent control functions, it is given for continuous-time systems, and it is closer in spirit to the definition of topological entropy in metric spaces due to Bowen [2] and Dinaburg [8] (note, however, that, due to the presence of time-dependent controls, these control systems are not dynamical systems.) In the present note we will show that these control entropies are essentially equivalent. We restrict this comparison to the discrete-time setting, since the analysis of feedbacks in the continuous-time case would presuppose more technicalities. Furthermore, we concentrate on the comparison between the feedback and the open-loop version, imposing a strong invariance 
condition.

In Section 2 we briefly recall the definitions of topological entropy for maps, and of invariance topological feedback entropy and invariance entropy for control systems. Section 3 presents the main result, equivalence between the considered control entropies, and Section 4 draws some conclusions and adds further comments.

Notation. The number of elements of a finite set $S$ (its cardinality) is denoted by $\# S$. We write log for the logarithm to the basis 2 .

2. Definitions of entropies. In this section, we briefly recall the concept of topological entropy in the theory of dynamical systems and discuss concepts of control entropies.

Consider a continuous map $f: X \rightarrow X$ on a compact topological space $X$, or the associated discrete-time system given by the iteration scheme

$$
x_{k+1}=f\left(x_{k}\right), \quad k \geq 0 .
$$

Adler, Konheim, and McAndrew [1] define the topological entropy of an open cover $\mathcal{U}$ of $X$ (i.e., $\mathcal{U}$ consists of open sets whose union is $X$ ) as

$$
H(\mathcal{U}):=\log \min \{\# \mathcal{V} \mid \mathcal{V} \text { a finite subcover of } \mathcal{U}\} .
$$

For two open covers $\mathcal{U}, \mathcal{V}$ of $X$, further open covers are defined by $\mathcal{U} \vee \mathcal{V}:=\{U \cap V \mid U \in$ $\mathcal{U}, V \in \mathcal{V}\}$ (called the join of $\mathcal{U}$ and $\mathcal{V}$ ) and $f^{-1}(\mathcal{U}):=\left\{f^{-1}(U) \mid U \in \mathcal{U}\right\}$. Then the topological entropy of $f$ with respect to an open cover $\mathcal{U}$ is

$$
h(f, \mathcal{U}):=\lim _{n \rightarrow \infty} \frac{1}{n} H\left(\bigvee_{i=0}^{n-1} f^{-i}(\mathcal{U})\right),
$$

and the topological entropy of $f$ is defined as

$$
h(f):=\sup \{h(f, \mathcal{U}) \mid \mathcal{U} \text { is an open cover of } X\} \in[0, \infty) \cup\{\infty\} .
$$

This number can be regarded as a measure for the fastest rate at which uncertainty about the initial state is reduced by iterating the map $f$ and tracking its trajectories with respect to an open cover (that is, one can only distinguish between two points if they lie in different elements of the open cover). Topological entropy has been constructed in strict analogy to the measure-theoretic entropy due to Kolmogorov and Sinai, where for an invariant measure $\mu$ instead of open covers measurable partitions $\xi=\left\{C_{\alpha}\right\}_{\alpha \in A}$ are taken and and $H(\xi):=-\sum_{\alpha} \mu\left(C_{\alpha}\right) \log \mu\left(C_{\alpha}\right)$; see, e.g., Katok and Hasselblatt [10, Chapter 4], Downarowicz [9], or Walters [19].

An equivalent definition of topological entropy for a continuous map $f$ on a compact metric space $(X, d)$, due to Bowen [2] and Dinaburg [8], is the following. For $\varepsilon>0$ and $\tau \in \mathbb{N}$ a set $S \subset X$ is called $(\tau, \varepsilon)$-spanning, if for every $x \in X$ there is $y \in S$ with $d\left(f^{i}(x), f^{i}(y)\right)<\varepsilon$ for $i=0,1, \ldots, \tau-1$. Then let $s(f, \tau, \varepsilon)$ be the minimal cardinality of such a set and define

$$
h_{\mathrm{span}}(f):=\lim _{\varepsilon \rightarrow 0} \limsup _{\tau \rightarrow \infty} \frac{1}{\tau} \log s(f, \tau, \varepsilon) .
$$

These definitions yield the same value. They present a way to measure the information about the initial state generated by the iterates of the map $f$.

We note that also for noncompact spaces, the Bowen-Dinaburg definition applies by considering compact sets of initial values and then taking the supremum over all 
compact sets, see Bowen [2]. A generalization of the open-cover definition to certain maps on noncompact spaces has recently been given by Patrão [17]. This, however, may give results which differ from Bowen's version, in particular for linear maps on $\mathbb{R}^{n}$.

Next recall topological feedback entropy as defined by Nair, Evans, Mareels, and Moran [16]. Let $X$ be a topological space and consider a control system on $X$ of the form

$$
x_{k+1}=F\left(x_{k}, u_{k}\right)=F_{u_{k}}\left(x_{k}\right), \quad k \geq 0,
$$

where $F: X \times U \rightarrow X$, and $U$ is an arbitrary set. Further assume that $F_{u}:=F(\cdot, u)$ : $X \rightarrow X$ is continuous for each $u \in U$. For a sequence $\underline{u}=\left(u_{0}, u_{1}, \ldots\right)$ of controls in $U$ we write

$$
\varphi: \mathbb{N}_{0} \times X \times U^{\mathbb{N}_{0}} \rightarrow X, \quad \varphi(k, x, \underline{u}):=F_{u_{k-1}} \circ \cdots \circ F_{u_{0}}(x) .
$$

Here $U^{\mathbb{N}_{0}}$ is the set of all functions from $\mathbb{N}_{0}:=\mathbb{N} \cup\{0\}$ to $U$. Where convenient, we use the notation $\varphi(k, x, \underline{u})$ also if $\underline{u}$ is only defined on a finite interval $\{0, \ldots, \tau\}$ with $\tau+1 \geq k$. Note that all maps $\varphi_{k, u}(\cdot):=\varphi(k, \cdot, \underline{u})$ on $X$ are continuous.

Let $Q \subset X$ be a compact set with nonvoid interior denoted by int $Q$ which fulfills the following strong invariance condition (essentially, this is assumption SI in [16, p. 1586]):

For every $x \in Q$ there is $u_{x} \in U$ with $F\left(x, u_{x}\right) \in \operatorname{int} Q$.

Let $\mathcal{A}$ be an open cover of $Q, \tau$ a positive integer, and $G: \mathcal{A} \rightarrow U^{\tau}$ a map with components $G_{0}, \ldots, G_{\tau-1}$ that assign control values to all sets in $\mathcal{A}$, such that for every $A \in \mathcal{A}$ the sequence of controls $G(A)$ yields $\varphi(k, A, G(A)) \subset \operatorname{int} Q$ for all $k \in\{1, \ldots, \tau\}$. Then we call the triple $(\mathcal{A}, \tau, G)$ an invariant open cover of $Q$. The existence of an invariant open cover easily follows from the strong invariance assumption on $Q$ and the continuity assumption for the maps $F_{u}, u \in U$. Now, for any sequence $\alpha:=\left(A_{i}\right)_{i \geq 0}$ of sets in $\mathcal{A}$ we define the control sequence

$$
\underline{u}(\alpha):=\left(u_{0}, u_{1}, \ldots\right) \text { with }\left(u_{l}\right)_{l=(i-1) \tau}^{i \tau-1}=G\left(A_{i-1}\right) \text { for all } i \geq 0 .
$$

We further define for each $j \geq 1$ the set

$$
B_{j}(\alpha):=\left\{x \in X \mid \varphi(i \tau, x, \underline{u}(\alpha)) \in A_{i} \text { for } i=0,1, \ldots, j-1\right\} .
$$

Then $B_{j}(\alpha)$ is an open set, since it can be written as the finite intersection of preimages of open sets under continuous mappings,

$$
B_{j}(\alpha)=\bigcap_{i=0}^{j-1}\left\{x \in X \mid \varphi(i \tau, x, \underline{u}(\alpha)) \in A_{i}\right\}=\bigcap_{i=0}^{j-1} \varphi_{i \tau, \underline{u}(\alpha)}^{-1}\left(A_{i}\right) .
$$

Furthermore, for each $j \geq 1$, letting $\alpha$ run through all sequences of elements in $\mathcal{A}$, the family

$$
\mathcal{B}_{j}:=\left\{B_{j}(\alpha) \mid \alpha \in \mathcal{A}^{\mathbb{N}_{0}}\right\}
$$

is an open cover of $Q$. Using the join operation for open covers, introduced above, we can express $\mathcal{B}_{j}$ also by

$$
\mathcal{B}_{j}=\mathcal{A} \vee \varphi_{\tau, \underline{u}(\alpha)}^{-1}(\mathcal{A}) \vee \ldots \vee \varphi_{(j-1) \tau, \underline{u}(\alpha)}^{-1}(\mathcal{A})
$$


Let $N\left(\mathcal{B}_{j} \mid Q\right)$ denote the minimal number of elements in a finite subcover of $\mathcal{B}_{j}$, and define the invariance topological feedback entropy $h_{\mathrm{fb}}(Q)$ by

$$
h_{\mathrm{fb}}(\mathcal{A}, \tau, G):=\lim _{j \rightarrow \infty} \frac{1}{j \tau} \log N\left(\mathcal{B}_{j} \mid Q\right), \quad h_{\mathrm{fb}}(Q):=\inf _{(\mathcal{A}, \tau, G)} h_{\mathrm{fb}}(\mathcal{A}, \tau, G),
$$

where the infimum is taken over all invariant open covers. Existence of the limit follows from subadditivity of the sequence $j \mapsto \log N\left(\mathcal{B}_{j} \mid Q\right)$ and the following elementary subadditivity lemma which we state and prove for completeness.

LEMMA 2.1. Let $\left(a_{n}\right)_{n \in \mathbb{N}}$ be a subadditive sequence of real numbers, i.e., $a_{m+n} \leq$ $a_{m}+a_{n}$ for all $m, n \in \mathbb{N}$. Then $a_{n} / n$ converges (the limit may be $-\infty$ ), and

$$
\lim _{n \rightarrow \infty} \frac{a_{n}}{n}=\inf _{n \in \mathbb{N}} \frac{a_{n}}{n}=: \gamma
$$

Proof. Fix $N \in \mathbb{N}$ and write $n=k(n) N+r(n)$ with $k(n) \in \mathbb{N}_{0}$ and $0 \leq r(n)<N$, hence $k(n) / n \rightarrow 1 / N$ for $n \rightarrow \infty$. Clearly, $a_{k}$ is bounded for $0 \leq k<N$ for any $N$. By subadditivity, for any $n \in \mathbb{N}$

$$
\gamma \leq \frac{a_{n}}{n} \leq \frac{1}{n}\left[a_{k(n) N}+a_{r(n)}\right] \leq \frac{1}{n}\left[k(n) a_{N}+a_{r(n)}\right] .
$$

Hence, for $\varepsilon>0$ there exists an $N_{0}(\varepsilon, N) \in \mathbb{N}$ such that for all $n>N_{0}(\varepsilon, N)$

$$
\gamma \leq \frac{a_{n}}{n} \leq \frac{a_{N}}{N}+\varepsilon
$$

Since $\varepsilon$ and $N$ are arbitrary, this implies

$$
\gamma \leq \liminf _{n \rightarrow \infty} \frac{a_{n}}{n} \leq \limsup _{n \rightarrow \infty} \frac{a_{n}}{n} \leq \gamma,
$$

which concludes the proof.

Comparing the definition of topological feedback entropy in (2.4) to the topological entropy of maps, one realizes that it appears as a natural generalization, where, instead of a single map, all combinations of maps generated by feedback are considered. Then the infimum is taken, since, in contrast to the theory of dynamical systems, not the maximal amount of information generated by the system is of interest, but the minimal amount of information needed for making the subset $Q$ invariant.

We turn to the definition of invariance entropy as in Colonius and Kawan [4], adapted to the setting above. For a natural number $\tau \in \mathbb{N}$, a set $\mathcal{S} \subset U^{\tau}$ is called $(\tau, Q)$-spanning if for every $x \in Q$ there is $\underline{u} \in \mathcal{S}$ such that $\varphi(j, x, \underline{u}) \in \operatorname{int} Q$ for all $j \in\{1, \ldots, \tau\}$. The minimal cardinality of such a set is denoted by $r_{\text {inv }}(\tau, Q)$ and we define the invariance entropy of $Q$ by

$$
h_{\mathrm{inv}}(Q):=\limsup _{\tau \rightarrow \infty} \frac{1}{\tau} \log r_{\mathrm{inv}}(\tau, Q) .
$$

The following proposition shows that this number is finite and the limit superior is in fact a limit.

Proposition 2.2. Assume that the strong invariance condition is satisfied. Then $r_{\text {inv }}(\tau, Q)<\infty$ for all $\tau>0$ and

$$
h_{\mathrm{inv}}(Q)=\lim _{\tau \rightarrow \infty} \frac{1}{\tau} \log r_{\mathrm{inv}}(\tau, Q)=\inf _{\tau \geq 1} \frac{1}{\tau} \log r_{\mathrm{inv}}(\tau, Q) .
$$


Proof. Let $\tau \geq 1$ and pick an arbitrary $x \in Q$. By strong invariance, we find $u_{1}, \ldots, u_{\tau} \in U$ with $x_{j}:=\varphi\left(j, x,\left(u_{1}, \ldots, u_{\tau}\right)\right) \in \operatorname{int} Q$ for all $j$. Each $x_{j}$ has an open neighborhood $V_{j} \subset \operatorname{int} Q$. By continuity, we find an open neighborhood $W_{x}$ of $x$ with $\varphi\left(j, W_{x},\left(u_{1}, \ldots, u_{\tau}\right)\right) \subset V_{j}$ for $j=1, \ldots, \tau$. By compactness of $Q$, finitely many of such neighborhoods are sufficient to cover $Q$. The corresponding control sequences form a finite $(\tau, Q)$-spanning set. To show (2.6), we apply the subadditivity lemma 2.1 to the sequence $\tau \mapsto \log r_{\text {inv }}(\tau, Q)$. In order to show subadditivity, consider a $\left(\tau_{1}, Q\right)$-spanning set $\mathcal{S}_{1}$ and a $\left(\tau_{2}, Q\right)$-spanning set $\mathcal{S}_{2}$. Then define control sequences of length $\tau_{1}+\tau_{2}$ by

$$
\underline{w}:=\left(u_{0}, \ldots, u_{\tau_{1}-1}, v_{0}, \ldots, v_{\tau_{2}-1}\right) \in U^{\tau_{1}+\tau_{2}}
$$

for each $\underline{u}:=\left(u_{0}, \ldots, u_{\tau_{1}-1}\right) \in \mathcal{S}_{1}$ and $\underline{v}:=\left(v_{0}, \ldots, v_{\tau_{2}-1}\right) \in \mathcal{S}_{2}$. The set of all such control sequences $\underline{w}$ is a $\left(\tau_{1}+\tau_{2}\right)$-spanning set of cardinality $\# S_{1} \cdot \# \mathcal{S}_{2}$, which implies $\log r_{\text {inv }}\left(\tau_{1}+\tau_{2}, Q\right) \leq \log r_{\text {inv }}\left(\tau_{1}, Q\right)+\log r_{\text {inv }}\left(\tau_{2}, Q\right) . \square$

This definition of invariance entropy is closer in spirit to the Bowen-Dinaburg definition of topological entropy, since spanning sets are used; here they are sets of control functions, not of initial states, and it is not required that trajectories remain close up to time $\tau$, but it is required that they remain in $Q$.

3. Equivalence between entropies. In the following, we show that the invariance topological feedback entropy (2.4) and the invariance entropy (2.5) based on feedbacks and on open-loop controls, respectively, coincide.

THEOREM 3.1. Let $Q \subset X$ be a compact subset that satisfies the strong invariance condition for control system $(2.1)$. Then $h_{\mathrm{fb}}(Q)=h_{\mathrm{inv}}(Q)$.

Proof. For a fixed $\tau \geq 1$, let $\mathcal{S}$ be a minimal $(\tau, Q)$-spanning set. Define for $\underline{u} \in \mathcal{S}$

$$
A(\underline{u}):=\{x \in Q \mid \varphi(j, x, u) \in \operatorname{int} Q \text { for } j=1, \ldots, \tau\} .
$$

It is clear that the sets $A(\underline{u})$ form an open cover $\mathcal{A}$ of $Q$. Now define $\tau$ maps $G_{k}$ : $\mathcal{A} \rightarrow U$ by

$$
G_{k}(A(\underline{u})):=u_{k}, \quad k=0, \ldots, \tau-1 .
$$

Since $\mathcal{S}$ is minimal, this definition makes sense. Clearly, $(\mathcal{A}, \tau, G)$ is an invariant open cover of $Q$. We have the trivial inequality $\# \mathcal{B}_{j} \leq(\# \mathcal{A})^{j}$ (cf. $(2.3)$ ), which implies

$$
h_{\mathrm{fb}}(Q) \leq \lim _{j \rightarrow \infty} \frac{\log N\left(\mathcal{B}_{j} \mid Q\right)}{j \tau} \leq \frac{\log \# \mathcal{A}}{\tau}=\frac{1}{\tau} \log r_{\mathrm{inv}}(\tau, Q) .
$$

Since this holds for each $\tau \geq 1$, we obtain $h_{\mathrm{fb}}(Q) \leq h_{\text {inv }}(Q)$. To show the converse inequality, let $(\mathcal{A}, \tau, G)$ be an invariant open cover of $Q$. Choosing a finite subcover $\mathcal{A}^{\prime}$ of $\mathcal{A}$ and restricting the functions $G_{k}$ to that subcover, we obtain another invariant open cover $\left(\mathcal{A}^{\prime}, \tau, G^{\prime}\right)$ such that $h_{\mathrm{fb}}\left(\mathcal{A}^{\prime}, \tau, G^{\prime}\right) \leq h_{\mathrm{fb}}(\mathcal{A}, \tau, G)$. Therefore, we may assume that $\mathcal{A}$ is finite. Then we can construct a $(j \tau, Q)$-spanning set $\mathcal{S}_{j}$ for each $j \geq 1$ with $N\left(\mathcal{B}_{j} \mid Q\right)$ elements as follows: Let $\tilde{\mathcal{B}}_{j}$ be a minimal subcover of $\mathcal{B}_{j}$. Each element of $\tilde{\mathcal{B}}_{j}$ corresponds to a particular sequence of elements in $\mathcal{A}$ and an associated control sequence $\underline{u}(\alpha)$ as defined in (2.2). The set of these control sequences obviously forms a $(j \tau, Q)$-spanning set. Hence, we obtain

$$
r_{\text {inv }}(j \tau, Q) \leq N\left(\mathcal{B}_{j} \mid Q\right) \text { for all } j \geq 1,
$$


implying

$$
h_{\mathrm{inv}}(Q)=\lim _{j \rightarrow \infty} \frac{1}{j \tau} \log r_{\mathrm{inv}}(j \tau, Q) \leq \lim _{j \rightarrow \infty} \frac{1}{j \tau} \log N\left(\mathcal{B}_{j} \mid Q\right)=h_{\mathrm{fb}}(\mathcal{A}, \tau, G) .
$$

Since this holds for every $(\mathcal{A}, \tau, G)$, the desired inequality follows. $\square$

We finish the paper with a simple example.

EXAMPLE 3.2. Consider a scalar linear control system given by

$$
x_{k+1}=a x_{k}+u_{k}, \quad u_{k} \in U:=[-1-\varepsilon, 1+\varepsilon],
$$

for some $\varepsilon>0$ and $a>1$. We claim that the compact interval $Q:=\left[-\frac{1}{a-1}, \frac{1}{a-1}\right]$ satisfies the strong invariance condition and that its entropy is given by

$$
h_{\mathrm{fb}}(Q)=h_{\mathrm{inv}}(Q)=\log a .
$$

Indeed, take some $x_{0} \in Q$ and let $u_{0}:=(1-a) x_{0} \pm \delta$ with $0<\delta \leq \varepsilon$. Then

$$
-1-\varepsilon \leq(1-a) x_{0} \pm \delta \leq 1+\varepsilon
$$

Hence, $u_{0}$ is an admissible control value, and putting it into equation (3.1) we obtain

$$
x_{1}:=a x_{0}+(1-a) x_{0} \pm \delta=x_{0} \pm \delta .
$$

This implies that every initial state in $Q$ can be steered into the interior of $Q$ in one time step, showing strong invariance of $Q$.

Now, let us compute the entropy of $Q$. To show that $h_{\mathrm{inv}}(Q) \geq \log a$, we use a volume argument for the Lebesgue measure $\lambda$. Let $\mathcal{S}$ be a minimal $(\tau, Q)$-spanning set and define

$$
Q_{\underline{u}}:=\{x \in Q: \varphi(j, x, \underline{u}) \in \operatorname{int} Q \text { for } j=1, \ldots, \tau\}, \quad \underline{u} \in \mathcal{S} .
$$

By definition of spanning sets, $Q=\bigcup_{\underline{u} \in \mathcal{S}} Q_{\underline{u}}$. Since $\varphi(\tau, \cdot, \underline{u})$ maps $Q_{\underline{u}}$ into int $Q$, it holds that

$$
\lambda\left(\varphi\left(\tau, Q_{\underline{u}}, \underline{u}\right)\right)=a^{\tau} \lambda\left(Q_{\underline{u}}\right) \leq \lambda(\operatorname{int} Q),
$$

which implies

$$
\lambda(\operatorname{int} Q) \leq \sum_{\underline{u} \in \mathcal{S}} \lambda\left(Q_{\underline{u}}\right) \leq \# \mathcal{S} \cdot \max _{\underline{u} \in \mathcal{S}} \lambda\left(Q_{\underline{u}}\right) \leq \# \mathcal{S} \cdot \frac{\lambda(\operatorname{int} Q)}{a^{\tau}} .
$$

Hence, we obtain $r_{\mathrm{inv}}(\tau, Q) \geq a^{\tau}$ for all $\tau$ which yields the desired estimate.

To show the upper estimate, we explicitly construct $(\tau, Q)$-spanning sets. First note that the transition map $\varphi$ for constant control sequences $\underline{u}=(u, u, \ldots)$ is

$$
\varphi(\tau, x, \underline{u})=a^{\tau} x+\sum_{i=0}^{\tau-1} a^{\tau-1-i} u=a^{\tau}\left(x+\frac{u}{a-1}\right)-\frac{u}{a-1} .
$$

Now we construct $\left(\tau_{k}, Q\right)$-spanning sets for the times

$$
\tau_{k}:=\left\lfloor\frac{k}{\log a}\right\rfloor-1, \quad k \geq k_{0},
$$


where $k_{0}$ is chosen large enough so that $\left\lfloor k_{0} / \log a\right\rfloor \geq 2$. Note that

$$
a^{\tau_{k}}<a^{\frac{k}{\log a}}=\left(2^{\log a}\right)^{\frac{k}{\log a}}=2^{k} .
$$

For each $k \geq 1$ we subdivide $Q$ into $2^{k}$ subintervals of the same length:

$$
Q_{j}:=-\frac{1}{a-1}+\frac{2}{a-1}\left[\frac{j}{2^{k}}, \frac{j+1}{2^{k}}\right], \quad j=0,1, \ldots, 2^{k}-1 .
$$

Then we associate to each $Q_{j}$ with $j \notin\left\{0,2^{k}-1\right\}$ a constant control sequence defined by

$$
\underline{u}_{j}:=\left(u_{j}, u_{j}, \ldots, u_{j}\right) \in U^{\tau}, \quad u_{j}:=1-\frac{2 j}{2^{k}-1} \in[-1,1] \subset U .
$$

For $j=0$ and $j=2^{k}-1$ we use the control values $u_{0}:=1+\delta$ and $u_{2^{k}-1}:=-1-\delta$, respectively, where $\delta>0$ is chosen sufficiently small.

Now we apply the control sequence $\underline{u}_{j}$ to the interval $Q_{j}$. We use the abbreviation $b:=a-1$ and first assume that $j \notin\left\{0,2^{k}-1\right\}$. Then for each $t \in\left\{1, \ldots, \tau_{k}\right\}$, using (3.2), we obtain

$$
\begin{aligned}
\varphi\left(t,-\frac{1}{b}+\frac{2}{b} \frac{j}{2^{k}}, \underline{u}_{j}\right) & =\frac{1}{b}\left(2 j a^{t}\left(\frac{1}{2^{k}}-\frac{1}{2^{k}-1}\right)-\left(1-\frac{2 j}{2^{k}-1}\right)\right) \\
& \geq \frac{1}{b}\left(2 j a^{\tau_{k}}\left(\frac{1}{2^{k}}-\frac{1}{2^{k}-1}\right)-\left(1-\frac{2 j}{2^{k}-1}\right)\right) \\
& \stackrel{(3.3)}{ } \frac{1}{b}\left(2 j 2^{k}\left(\frac{1}{2^{k}}-\frac{1}{2^{k}-1}\right)-\left(1-\frac{2 j}{2^{k}-1}\right)\right) \\
& =\frac{1}{b}\left(-\frac{2 j}{2^{k}-1}-\left(1-\frac{2 j}{2^{k}-1}\right)\right)=-\frac{1}{b},
\end{aligned}
$$

where we used that $1 / 2^{k}-1 /\left(2^{k}-1\right)<0$. For $j=0$ we obtain

$$
\varphi\left(t,-\frac{1}{b}, \underline{u}_{0}\right)=a^{t} \frac{\delta}{b}-\frac{1+\delta}{b}=-\frac{1}{b}\left(1-\left(a^{t}-1\right) \delta\right)>-\frac{1}{b} .
$$

A similar estimate as above yields for $j \notin\left\{0,2^{k}-1\right\}$

$$
\varphi\left(t,-\frac{1}{b}+\frac{2}{b} \frac{j+1}{2^{k}}, \underline{u}_{j}\right)<\frac{1}{b}
$$

and for $j=2^{k}-1$

$$
\varphi\left(t, \frac{1}{b}, \underline{u}_{2^{k}-1}\right)=a^{t} \frac{-\delta}{b}+\frac{1+\delta}{b}=\frac{1}{b}\left(1-\left(a^{t}-1\right) \delta\right)<\frac{1}{b} .
$$

Note that the corresponding estimates for the right endpoint of $Q_{0}$ and the left endpoint of $Q_{2^{k}-1}$ are trivial. Since for each $t, \varphi(t, \cdot, \underline{u})$ maps $Q_{j}$ onto a compact interval without reversing the left and right endpoints, we have shown that the set $\mathcal{S}_{k}:=$ $\left\{\underline{u}_{0}, \ldots, \underline{u}_{2^{k}-1}\right\}$ is $\left(\tau_{k}, Q\right)$-spanning, which implies

$$
r_{\text {inv }}\left(\tau_{k}, Q\right) \leq 2^{k} \quad \text { for all } k \geq 1
$$


This gives

$$
\limsup _{k \rightarrow \infty} \frac{1}{\tau_{k}} \log r_{\text {inv }}\left(\tau_{k}, Q\right) \leq \limsup _{k \rightarrow \infty} \frac{k}{\frac{k}{\log a}-2}=\log a .
$$

If $\left(m_{n}\right)_{n \geq 1}$ is an arbitrary sequence of integers with $m_{n} \rightarrow \infty$, for each $n$ let $k_{n}$ be the minimal integer such that $m_{n} \leq\left\lfloor k_{n} / \log a\right\rfloor-1$. Then $\left\lfloor\left(k_{n}-1\right) / \log a\right\rfloor-1 \leq m_{n}$. This implies

$$
m_{n} \geq \frac{k_{n}-1}{\log a}-1=\frac{k_{n}}{\log a}-\underbrace{\left(\frac{1}{\log a}+1\right)}_{=: d} \geq\left\lfloor\frac{k_{n}}{\log a}\right\rfloor-d .
$$

Using monotonicity of $\tau \mapsto r_{\mathrm{inv}}(\tau, Q)$, this yields

$$
\begin{aligned}
\limsup _{n \rightarrow \infty} \frac{1}{m_{n}} \log r_{\text {inv }}\left(m_{n}, Q\right) & \leq \limsup _{n \rightarrow \infty} \frac{1}{\left\lfloor\frac{k_{n}}{\log a}\right\rfloor-d} \log r_{\text {inv }}\left(\left\lfloor\frac{k_{n}}{\log a}\right\rfloor-1, Q\right) \\
& =\limsup _{n \rightarrow \infty} \frac{1}{\tau_{k_{n}}} \log r_{\text {inv }}\left(\tau_{k_{n}}, Q\right) \stackrel{(3.4)}{\leq} \log a .
\end{aligned}
$$

Hence, we have proved that $\log a$ is an upper bound for $h_{\mathrm{inv}}(Q)$.

There are many variants of the definitions above which also make sense. For example, in the definition of strong invariance, one can fix a compact set $Q^{\prime}$ in the interior of $Q$ and require that $\varphi(j, A, G(A)) \subset \operatorname{int} Q^{\prime}$. Or one may add a further compact set $K \subset Q$ of initial values and then require that one remains within $Q$ and, finally reaches $Q^{\prime} \subset \operatorname{int} Q$. In all of these cases, the arguments above show that the feedback definition and the open-loop definition coincide.

Changing the definitions slightly, the strong invariance condition may be weakened by assuming just invariance of $Q$ (i.e., for every $x \in Q$ there is $u_{x} \in U$ with $F\left(x, u_{x}\right) \in Q$.) Then one may obtain existence of finite spanning sets by allowing that the corresponding trajectories are within $\varepsilon$-neighborhoods of $Q$, and then, after taking the limit superior for time tending to $\infty$ letting $\varepsilon \rightarrow 0$ (see [4, Section 3]).

4. Conclusions. For discrete-time systems, the discussion in Section 3 has shown that the two considered notions of entropy for the problem to render a compact subset of the state space invariant are equivalent, provided that the strong invariance condition is satisfied. Either, one may consider the growth rate of the number of subsets (in an open cover) where the feedback law is constant when time goes to infinity; or one may consider the growth rate of the number of different open-loop controls as time tends to infinity. We also remark that for continuous-time systems it seems easier to use the second notion which avoids the discussion of appropriate regularity properties of feedbacks.

\section{REFERENCES}

[1] R. Adler, A. Konheim, and M. McAndrew, Topological entropy, Trans. Amer. Math. Soc., 114 (1965), pp. 61-85.

[2] R. Bowen, Entropy for group endomorphisms and homogeneous spaces, Trans. Amer. Math. Soc., 153 (1971). Erratum, 181(1973), pp. 509-510.

[3] F. Colonius And U. Helmke, Entropy of controlled invariant subspaces, Zeitschrift für Angewandte Mathematik und Mechanik, (2011). submitted. 
[4] F. Colonius and C. Kawan, Invariance entropy for control systems, SIAM J. Control Optim., 48 (2009), pp. 1701-1721.

[5] _ Invariance entropy for outputs, Mathematics of Control, Signals, and Systems, 22(3) (2011), pp. 203-227.

[6] A. Da Silva, Invariance entropy for random control systems, to appear in: Math. Control Signals Systems, 2013.

[7] C. DE PeRsis, $n$-bit stabilization of $n$-dimensional nonlinear systems in feedforward form, IEEE Trans. Autom. Control 50, No. 3 (2005), pp. 299-311.

[8] E. Dinaburg, On the relations among various entropy characteristics of dynamical systems, Math. USSR-Izvestija, 5 (1971), pp. 337-378.

[9] T. Downarowicz, Entropy in Dynamical Systems, New Mathematical Monographs, 18. Cambridge University Press, Cambridge, 2011.

[10] A. Katok and B. Hasselblatt, Introduction to the Modern Theory of Dynamical Systems, Cambridge University Press, 1995.

[11] C. KaWAN, Invariance entropy of control systems. Doctoral thesis, Institut für Mathematik, Universität Augsburg, 2009.

[12] — Invariance entropy of control sets, SIAM J. Control Optim., 49 (2011), pp. 732-751.

[13] _ L L Lower bounds for the strict invariance entropy, Nonlinearity, 24 (2011), pp. 1909-1935.

[14] —, Upper and lower estimates for invariance entropy, Discrete Contin. Dyn. Syst., 30 (2011), pp. 169-186.

[15] — Minimal Data Rates for Invariance of Sets. An Introduction to Invariance Entropy for Finite-Dimensional Deterministic Systems, forthcoming monograph.

[16] G. Nair, R. J. Evans, I. Mareels, And W. Moran, Topological feedback entropy and nonlinear stabilization, IEEE Trans. Aut. Control, 49 (2004), pp. 1585-1597.

[17] M. PatrÃO, Entropy and its variational principle for non-compact metric spaces, Ergod. Th. \& Dynam. Sys., 30 (2010), pp. 1529-1542.

[18] A. V. SAVkin, Analysis and synthesis of networked control systems: topological entropy, observability, robustness and optimal control, Automatica, Vol. 42 (2006), pp. 51-62.

[19] P. Walters, An Introduction to Ergodic Theory, Graduate Texts in Mathematics, 79. SpringerVerlag, New York-Berlin, 1982.

[20] W. S. WONG AND R. W. Brockett, Systems with finite communication bandwidth constraintsII: stabilization with limited information feedback, IEEE Trans. Autom. Control 44, No. 5 (1999), pp. 1049-1053. 